NOTICE: This is the author's version of a work that was accepted for publication in Appetite. Changes resulting from the publishing process, such as peer review, editing, corrections, structural formatting, and other quality control mechanisms may not be reflected in this document. Changes may have been made to this work since it was submitted for publication. A definitive version was subsequently published in Appetite, Vol. 62 (2013). doi: 10.1016/j.appet.2012.11.021 
Predicting adolescent breakfast consumption in the UK and Australia using an extended theory of planned behaviour

\author{
Barbara Mullan $^{1 *}$, Cara Wong ${ }^{1} \&$ Emily Kothe ${ }^{1}$ \\ ${ }^{1}$ School of Psychology, University of Sydney \\ Sydney, NSW, Australia
}

Barbara Mullan (corresponding author)

Postal Address: School of Psychology, University of Sydney, Sydney, NSW, Australia, 2006

Email: barbara.mullan@sydney.edu.au

Phone: +61293516811

Cara Wong

Postal Address: School of Psychology, University of Sydney, Sydney, NSW, Australia, 2006

Email: cara.wong@sydney.edu.au

Emily Kothe

Postal Address: School of Psychology, University of Sydney, Sydney, NSW, Australia, 2006

Email: emily.kothe@ sydney.edu.au 


\begin{abstract}
The aim of this study was to investigate whether the Theory of Planned Behaviour (TPB) with the addition of risk awareness could predict breakfast consumption in a sample of adolescents from the UK and Australia. It was hypothesised that the TPB variables of attitudes, subjective norm and perceived behavioural control (PBC) would significantly predict intentions, and that inclusion of risk perception would increase the proportion of variance explained. Secondly it was hypothesised that intention and PBC would predict behaviour. Participants were recruited from secondary schools in Australia and the UK. A total of 613 participants completed the study (448 females, 165 males; mean $=14$ years \pm 1.1 ). The TPB predicted $42.2 \%$ of the variance in intentions to eat breakfast. All variables significantly predicted intention with PBC as the strongest component. The addition of risk made a small but significant contribution to the prediction of intention. Together intention and PBC predicted $57.8 \%$ of the variance in breakfast consumption.
\end{abstract}




\section{Predicting adolescent breakfast consumption in the UK and Australia using an extended theory of planned behaviour}

In the seminal 'Alameda 7' study (Belloc \& Breslow, 1972), eating breakfast was identified as one of the seven healthy habits that contributed to long term health and mortality. Despite this finding, research has found that the frequency of breakfast consumption has declined over time (Haines, Guilkey, \& Popkin, 1996), and breakfast skipping is particularly common in adolescents and young adults (Keski-Rahkonen, Kaprio, Rissanen, Virkkunen, \& Rose, 2003). Studies have shown that 12\% of Australian adolescents skip breakfast (Shaw, 1998), and 1 in 4 adolescents go to school hungry (Williams, 2005). In the UK, trends appear worse, where one study found that $19 \%$ of adolescents aged 11-16 regularly missed breakfast (Lattimore \& Halford, 2003), and a more recent study found that 39\% of girls and 27\% of boys aged 10-16 sometimes or always skipped breakfast (Sandercock et al, 2010).

Reviews of the health implications of breakfast skipping show a convincing link between breakfast consumption and nutritional adequacy (Rampersaud, Pereira, Girard, Adams, \& Metzl, 2005). Evidence from a number of studies suggests that individuals who consume breakfast are more likely to consume recommended quantities of important micronutrients such as calcium, iron, vitamins A and C, riboflavin, and zinc (Rampersaud et al., 2005). These micronutrients are important for healthy growth during adolescence and inadequate intakes have been linked to increased risk of disease and ill-health (Erdman, Macdonald, \& Zeisel, 2012). For example, low intake of calcium in adolescence, a frequently observed consequence of breakfast skipping, is associated with low peak bone density and higher risk of osteoporosis and fracture in older adulthood (Cashman, 2002; Matkovic, Fontana, Tominac, Goel, \& Chesnut, 1990; NIH 
Consensus Panel, 1994). Worryingly, nutrient deficits associated with breakfast skipping do not appear to be compensated for by changes in diet throughout the rest of the day (Rampersaud et al., 2005).

Skipping breakfast has also been shown to have deleterious effects on cognitive and academic performance. A study in India found that children aged 11-13 who ate a regular breakfast had significantly better immediate recall memory and higher grades than those children who did not (Gajre, Fernandez, Balakrishna, \& Vazir, 2010). Similarly, experimental studies have shown differences in cognitive performance between children who did or did not consume breakfast on the morning of the study (Wesnes, Pincock, Richardson, Helm, \& Hails, 2003). In a review of the literature, Rampersaud et al. (2005) showed that breakfast consumption was related to improved memory, test grades, and school attendance in children and adolescents.

The numerous positive health and social risks of skipping breakfast highlight the need for research to understand the personal and motivational factors affecting regular breakfast consumption in adolescents. A number of theoretical models have been used to predict health behaviours. One of the most dominant and commonly used models is the Theory of Planned Behaviour (TPB; Ajzen, 1991). According to the model, behavioural intention is the most proximal antecedent to behaviour. In general, the stronger the intention to engage in a behaviour, the more likely it will be performed. The TPB includes three independent predictors of behavioural intention; attitude (favourable or unfavourable evaluations about the behaviour); subjective norm (perceived social pressure to perform behaviour); and PBC (an individual's perceptions of the ease or difficulty of performing the behaviour of interest). While attitude and subjective norm are thought to influence behaviour through intention, $\mathrm{PBC}$ is argued to directly 
influence both intention and behaviour, particularly in situations where behaviour is not under the total control of the individual (Azjen, 1991).

The TPB has been shown to successfully predict intention and behaviour in a number of dietary behaviours (Berg, Jonsson, \& Conner, 2000; Collins \& Mullan, 2011; Conner, Norman, \& Bell, 2002; Fila \& Smith, 2006; Kothe, Mullan, \& Butow, In Press; Sainsbury \& Mullan, 2011; Seo, Lee, \& Nam, 2011; Verbeke \& Vackier, 2005; White, Terry, Troup, Rempel, \& Norman, 2010). The TPB has also been shown to be relatively strong in predicting breakfast skipping in young adult populations (Kothe, Amaratunga, \& Mullan, 2011; Wong \& Mullan, 2009). Specifically, Wong and Mullan (2009) found that the TPB predicted $64 \%$ of the variance in breakfast consumption at one week follow-up. Kothe et al (2011) found that the TPB predicted $41.5 \%$ of the variance in behaviour to consume breakfast at one month follow-up. Although no direct applications of the TPB to breakfast in children have been reported one study found that the TPB variables significantly predicted choices of milk and bread at breakfast time in a sample of Swedish children aged 11-15 years (Berg et al., 2000).

Although studies have confirmed the importance of the TPB variables in predicting intentions and the likelihood of future behaviour, there are usually large proportions of variance unaccounted for. A meta-analysis of the TPB found that over 161 studies, the TPB accounted for $27 \%$ of the variance in behaviour (Armitage \& Conner, 2001). Consequently the TPB can be criticised as an incomplete model and evidently other factors contribute in explaining intention and behaviour. Azjen (1991) acknowledged this and stated that the TPB is open to the inclusion of additional predictors if it can be shown that they capture a significant proportion of variance in behaviour after the theory's current variables are taken into account. One potential variable, is risk awareness. Several authors have criticised the theory of planned behaviour for failing to take 
risk awareness into account when seeking to understand intention to engage in a behaviour (Chorlton, Conner, \& Jamson, 2012; Conner, Kirk, Cade, \& Barrett, 2001; Norman \& Conner, 1996). Other models of health behaviour (e.g. the Health Action Process Approach and Protection Motivation Theory) would suggest that a minimum level of threat or concern is necessary to motivate to form intentions (Schwarzer et al., 2003).

There is some evidence that risk awareness may be important for developing a comprehensive model of breakfast consumption. In particular, recent research applying the Health Action Process Approach (Schwarzer et al., 2007) to the prediction of breakfast skipping in young adults found that risk awareness was a significant predictor of intention to skip breakfast (Mullan, Wong, Kothe, \& MacCann, 2012). Consistent with the Health Action Process Approach, the study conceptualised risk awareness as consisting of three major components: absolute risk, relative risk, and risk severity. However, while risk awareness was found to be an important predictor of intention to skip breakfast, the overall Health Action Process Approach model was found to be less effective than the Theory of Planned Behaviour at explaining breakfast consumption (Mullan et al., 2012). That research did not consider the predictive power of risk awareness once existing Theory of Planned Behaviour variables were taken into account. As such, it is unclear whether the addition of risk awareness would add to the prediction of breakfast over and above subjective norm, attitude, and perceived behavioural control. Research is needed to determine whether the observed relationship between risk awareness and intention remains once these variables are taken into account. Such research is especially important since there may be overlap between risk awareness and attitude since both constructs involve the evaluation of the likelihood of experiencing future consequences of behaviour and/or behaviour non-performance. While studies in other behavioural domains suggest that risk awareness is 
likely to be distinct from attitude (Bränström, Ullén, \& Brandberg, 2004), this research has not investigated the relative contribution of these variables to the prediction of intention.

The aim of the current study was to investigate whether the TPB, with the addition of risk awareness, could predict breakfast consumption in a sample of adolescents from the UK and Australia. It was hypothesised that the TPB variables of attitudes, subjective norm and PBC would significantly predict intentions, and that the addition of risk perception would increase the proportion of variance explained. Secondly it was hypothesised that intention and PBC would predict behaviour.

\section{Methods}

\section{Recruitment}

Participants were secondary school aged adolescents, recruited from schools in Australia and the UK from both urban and rural areas. Schools were found via school directories such as the National Education Directory, Australia; the Department of Education Science and Training; and the Schools Web Directory in the UK; Catholic School diocese listings (Australia only) and personal contacts. A variety of schools were initially contacted including private schools, Catholic schools, public schools, grammar schools, senior colleges and single sex and coeducation schools. These schools were from both urban and rural areas. Sampling of schools was stratified rather than random, however this sampling strategy was used to ensure that a wide variety of participants were sampled, to reduce potential biases that may occur from socioeconomic or cultural differences. This study was conducted according to the guidelines laid down in the Declaration of Helsinki and all procedures involving human participants were approved by the relevant ethics review panels. Written informed consent was obtained from all participants. 
Schools were first contacted by the researchers by email or telephone to ask if they were interested in participating in the study. Once the school had expressed interest in participating and a teacher had been specified as a liaison, each school was told they could choose which classes/years they thought were appropriate to participate as long as they fell within the age range of 11 to 18 . Initially, twenty-five schools agreed to participate; however, there was a high drop-out rate due to factors such as not enough time or failing to correspond with researchers after the initial agreement. Due to time and examination constraints of older students, the majority of schools agreed that students aged 14-15 could participate.

\section{Participating Schools}

Five schools from England participated in the study from a range of areas including Oxford, Worcester, Gloucester, Yorkshire and Hampshire. Four Australian schools participated from Brisbane, Queensland; Dubbo, New South Wales (NSW); Bathurst, NSW; and Hurstville, NSW.

\section{Questionnaires}

The TPB questionnaire was developed and informed by Fishbein and Ajzen's (1975) guidelines, and based on items used by Wong and Mullan (2009). The risk awareness measure was adapted from Schwarzer et al's Health Action Process Approach (2003).

Attitudes were assessed as the mean of 6 semantic differential scales (e.g. eating breakfast would be: bad-good, unnecessary-necessary, unpleasant-pleasant, unenjoyableenjoyable, harmful-beneficial, foolish-wise). Participants rated on a scale of 1-7 with a higher score indicating a more positive attitude. An alpha coefficient of .93 was reported $(\mathrm{M}=5.9, \mathrm{SD}$ $=1.3$, Median $=6.3$ ). 
Subjective norm was assessed by a single item "people who are important to me think I should eat breakfast'" (unlikely-likely), scored 1-7 with a higher score indicating more normative pressure $(\mathrm{M}=6.1, \mathrm{SD}=1.4$, Median $=7.0)$. The use of a single item to measure subjective norm is consistent with a number of previous studies using this construct to predict dietary behaviour (Armitage \& Conner, 2001; Mullan et al., 2012; Wong \& Mullan, 2009).

PBC was assessed as the mean of four, seven-point (1-7) items including two items for controllability (e.g. how much control over eating breakfast do you have?) and two for selfefficacy (e.g. I am confident I can eat breakfast). For this variable an alpha coefficient of .81 (M $=5.9, \mathrm{SD}=1.3$, Median $=6.5)$ was reported.

Risk awareness was measured with three risk components - absolute risk, relative risk and risk severity. Absolute risk was measured with three items (if you don't eat breakfast how do you estimate the likelihood that you will ever: suffer from less energy/have less concentration/ feel less healthy). This was measured on a 7 point Likert scale from very low to very high. A Cronbach's alpha coefficient of $.88(\mathrm{M}=15.2, \mathrm{SD}=4.89$, Median $=16.0)$ was reported. Relative risk was measured by asking participants, compared to other people of your age and sex, if you don't eat breakfast how do you estimate the likelihood that you will ever have less energy/have less concentration/ feel less healthy. An alpha coefficient of $.92(\mathrm{M}=14.4, \mathrm{SD}=4.71$, Median $=$ 15.0) was reported. The third component measured was risk severity (How severe would the following health related problems be for you: having less energy/less concentration/ feeling less healthy). These items were taken from a previous study which assessed risk awareness in the context of breakfast skipping behaviour in young adults using the Health Action Process Approach (Mullan et al., 2012). An alpha coefficient of $.89(\mathrm{M}=11.4, \mathrm{SD}=4.63$, Median $=$ 12.0) was reported in the current study. The combined effect of absolute, relative risk and risk 
severity had an alpha coefficient of .68. Due to the low internal consistency between the 3

measures of risk, the components were kept separate for analysis.

Behavioural intention was assessed as the mean of four items, each measured on sevenpoint scales (I intend/plan/aim/will make an effort to eat breakfast over the next 4 weeks). For Behavioural Intention the alpha coefficient was .97 $(\mathrm{M}=5.5, \mathrm{SD}=1.6$, Median $=6.5)$.

Behaviour was measured by asking participants how many times per week during the previous 4 weeks, they had eaten breakfast on a scale of 1-8 (never to 7 times a week). Breakfast eating was defined as 'consumption of a meal within 2 hours of waking'.

\section{Procedure}

Teachers from participating schools assisted the researchers in administering the online task by providing students with the relevant questionnaire URL and issuing individual participant IDs. Participants completed all questionnaires in one sitting.

\section{Analysis}

Data were analysed using SPSS version 15. Initial analyses were conducted to explore the data. Correlations and Multivariate Analysis of Variance (MANOVA) were used to determine whether there were country, age or gender differences in the data. Principal components factor analysis with Varimax rotation and Kaiser normalisation was used to ensure that key constructs were separate factors. The hypotheses were tested with a series of hierarchical linear multiple regressions.

\section{Results}

A total of 605 participants completed the study (448 females, 165 males) with a mean age of 14 years $(\mathrm{SD}=1.1$, range 11-18). There were 335 participants from Australia and 270 from the UK. The majority of participants indicated that they lived with their parents (97\%). See Table 1 for a 
breakdown of demographics by country. Eight-seven percent of students reported consuming breakfast on the day of the study.

Differences between gender and country and the main study variables [age, attitudes, subjective norms, $\mathrm{PBC}$, risk, intentions and behaviour regarding breakfast consumption] were explored using Multivariate Analysis of Variance (MANOVA). For country, significant differences were found for all variables except risk severity. On average, Australian participants were older than UK participants, had higher attitudes, subjective norm, PBC, absolute and relative risk awareness, intentions and self-reported behavior (See Table 2). For gender, females in the study were likely to be older, and hold more positive subjective norms than males. Males in the study scored higher on intention measures and were more likely to consume breakfast than females.

Bivariate correlations were calculated to explore the relationship between age, TPB variables, and risk variables (see Table 3). As shown in Table 3, age was only correlated with risk severity, such that risk severity decreased with increased age. Attitude, subjective norm, perceived behavioural control, relative risk, absolute risk, risk severity and intention were all significantly correlated with breakfast consumption frequency. Attitude, subjective norm, perceived behavioural control, relative risk, absolute risk, and risk severity were all positively correlated with intention. 
Factor analysis was used to further explore whether the three risk variables and attitude, subjective norm, and PBC from the TPB were distinct constructs. Using Principal Components Analysis, five factors emerged. These factors appeared above the 'elbow' on a Scree plot, had eigenvalues above one, and accounted for $75.6 \%$ of the variance in items. With Varimax rotation and Kaiser normalisation, items for the constructs of attitudes, perceived behavioural control, absolute risk, relative risk, and risk severity represented distinct factors (see Table 4). Subjective norm did not weigh strongly on any of the factors, however only one item was used to measure subjective norm.

\section{Predicting Intention}

Hierarchical linear multiple regression analyses for each country were conducted to analyse the predictive influence of each of the variables on intention (see Table 5). Attitude, subjective norm and PBC were entered in the first block and the three risk awareness scores in the second block. The analyses showed that overall the TPB was able to predict $27.6 \%$ of the variance in intentions to eat breakfast in the Australian sample. The three variables explained a larger proportion of variance in the UK sample (58.1\%). All variables made significant predictions to the prediction of intention, however, subjective norm was the weakest predictor. PBC was the strongest predictor of intentions only in the UK sample. The addition of the three risk components made a small but significant contribution to the variance explained for the UK participants $\left(\mathrm{R}^{2} \Delta=.028 ; \mathrm{F} \Delta_{3,263}=6.19, \mathrm{p}<.01\right)$. Only absolute risk, but not relative risk and risk severity, was a significant predictor of intentions. In the Australian group, the risk variables did not significantly increase the proportion of variance explained in intention $\left(\mathrm{R}^{2} \Delta=.016 ; \mathrm{F} \Delta_{3,328}=\right.$ $2.42, \mathrm{p}=.066$ ). However, relative risk significantly predicted intention (see Table 5).

\section{Predicting Behaviour}


Separate hierarchical regression analyses were conducted to analyse the predictive influence of each of the intention and PBC on breakfast consumption for the Australian and UK groups (see Table 6). The TPB predicted $59.0 \%$ of the variance in behaviour for the Australian adolescents, and $53.7 \%$ in the UK adolescents. Intentions were the strongest predictor of behaviour in both groups. However, $\mathrm{PBC}$ was only significant in the UK but not the Australian sample.

\section{Discussion}

The results of the current study investigated whether the TPB was a useful model in predicting intention and behaviour to consume breakfast in a large sample of adolescents in the UK and Australia. The addition of risk perception was also investigated, as previous studies have shown risk to be an important factor in the performance of health behaviours (Schwarzer et al, 2003). Importantly, risk was separated into three components - absolute, relative and severity, as reliability analyses showed these constructs were not unitary, and their independent contribution to intention was examined.

\section{Predicting Intention}

As hypothesised, across both countries all three TPB variables were found to be significant predictors of intentions to consume breakfast, however the proportion of variance varied between the countries. In Australia, the TPB variables predicted $27.6 \%$ of the variance in intention, whereas in the UK, the TPB variables predicted $58.1 \%$ of the variance in intention. This difference is striking, and may suggest that intention to consume breakfast is to a greater extent planned in the UK when compared to Australia. The strong prediction of intention in the UK sample is greater than reported in previous TPB studies. Very few cross cultural studies 
using the TPB have been reported so this important finding needs to be replicated and further explored.

PBC and attitudes were the strongest predictors of intention, supporting the utility of the TPB in explaining this behaviour. The relatively weak predictive power of the subjective norm construct may have been influenced by the use of single item measure of subjective norm. While the use of this type of measure is consistent with previous studies applying the TPB to breakfast consumption (Mullan et al., 2012; Wong \& Mullan, 2009), meta-analyses have shown that the use of single item measures of subjective norm may partially account for the weak predictive power of the construct (Armitage \& Conner, 2001). Nonetheless it may be that social norms are not important for this behaviour and future research is needed to explore this.

The addition of risk perception did not greatly enhance the predictive power of the study and lends support for the use of the TPB over models such as HAPA (which use risk measures). A recent study of breakfast in adults also found that the TPB was more useful that HAPA for breakfast eating (Mullan et al., 2012). Understanding the role of risk in health behaviour could benefit from qualitative research, which could disentangle the important components of risk to better inform quantitative research and interventions.

\section{Predicting Behaviour}

In line with the second hypothesis, the TPB was found to predict $59 \%$ and $54 \%$ of the variance in breakfast consumption in Australia and the UK respectively. This is comparable to previous breakfast research using the TPB (Kothe et al., 2012; Wong \& Mullan, 2009) supporting the use of the TPB for food behaviours in an adolescent cross-cultural population. Intention was the strongest predictor of behaviour, suggesting that breakfast consumption is mainly influenced by personal motivation. The finding that the theory better predicted breakfast 
consumption than intention to consume breakfast is consistent with previous applications of the model to breakfast consumption (Kothe et al., 2012; Wong \& Mullan, 2009). This pattern of results suggests that more work is still needed to determine the determinants of intention to consume breakfast.

However, the study also highlights the fact that not all intentions are translated into behaviour. This is consistent with a meta-analytic review that suggested that large increases in intention translate to only moderate changes in behaviour (Webb \& Sheeran, 2006). Future studies may investigate closing the intention-behaviour gap by including additional variables that may lie along the intention-behaviour continuum. For example, Wong and Mullan (2009) found that the inclusion of planning significantly moderated the intention-behaviour gap in young adult breakfast consumption. Self-regulatory abilities such as planning could be potentially useful for adolescent populations who are still developing self-regulatory and executive abilities (Koechlin, Ody, \& Kouneiher, 2003).

\section{Strengths and Limitations}

The study was the first to use the TPB to predict breakfast in an adolescent population in two developed countries. The study adds to the small but growing body of research showing that the TPB accounts for a large proportion of variance in breakfast eating frequency, and provides a better model for breakfast consumption than for intention to consume breakfast. The extension of this research into an adolescent population is a major strength of the current research. These findings are important for the development of interventions to target breakfast consumption, especially since most attempts to increase breakfast consumption are targeted at school children.

However, there are some limitations of the current study that should be taken into account when interpreting the current research. Firstly, because of practical constraints related to 
the collection of data in a school setting, this study used a cross-sectional design. It has been argued that cross-sectional designs may inflate the association between TPB variables and overestimate the predictive utility of the model. Future studies should investigate whether the relationships between intention, $\mathrm{PBC}$, and behaviour remain stable when behaviour is tested after a delay. Secondly, like most studies which have used the TPB to investigate dietary behaviour, the present study measured breakfast consumption using a self-report measure. While metaanalyses on the TPB have found that there were high correlations in the TPB prediction of both objectively measured data and self-report data (Armitage \& Conner, 2001), it would be interesting to determine whether the predictive utility of the model holds if breakfast consumption is measured objectively. Finally, the population were not as diverse as originally planned. Although a variety of schools were contacted from various regions, the majority of participants were from high SES backgrounds and Caucasian ethnicity. Breakfast consumption is known to vary according to sociodemographic factors - such that breakfast skipping is higher in lower SES groups (Mullan \& Singh, 2010). The relative sociodemographic homogeneity of the current sample may limit the extent to which findings from this study can be generalised to other populations. Future researchers may wish to specifically target lower SES groups in order to evaluate the use of the TPB to predict breakfast consumption in those populations.

\section{Conclusion}

In conclusion, the current study provides further support for the TPB in predicting breakfast consumption. The study was the first to use the TPB to predict breakfast in an adolescent population in two developed countries. Risk perception showed some influence over intentions; however, this was outweighed by the original TPB variables. Despite this, the study suggests that absolute risk is differentially predictive of intentions compared to risk severity or 
relative risk. Intentions remained the strongest predictor of behaviour suggesting that efforts to increase intentions through targeting attitudes, subjective norms and PBC may have a knock on effect in increasing breakfast consumption. 


\section{References}

Ajzen, I. (1991). The Theory of Planned Behaviour. Organizational Behavior and Human Decision Processes, 50, 179-211.

Armitage, C., \& Conner, M. (2001). Efficacy of the Theory of Planned Behaviour: A metaanalytic review. British Journal of Social Psychology, 40, 471-499.

Belloc, N. B., \& Breslow, L. (1972). Relationship of physical health status and health practices. Preventive medicine, 1(3), 409-421.

Berg, C., Jonsson, I., \& Conner, M. (2000). Understanding choice of milk and bread for breakfast among Swedish children aged 11-15 years: an application of the Theory of Planned Behaviour. Appetite, 34(1), 5-19.

Bränström, R., Ullén, H., \& Brandberg, Y. (2004). Attitudes, subjective norms and perception of behavioural control as predictors of sun-related behaviour in Swedish adults. Preventive Medicine, 39(5), 992-999.

Cashman, K. (2002). Calcium intake, calcium bioavailability and bone health. British journal of Nutrition, 87(S2), S169-S177.

Chorlton, K., Conner, M., \& Jamson, S. (2012). Identifying the psychological determinants of risky riding: An application of an extended Theory of Planned Behaviour. Accident Analysis \&amp; Prevention, 49(0), 142-153.

Collins, A., \& Mullan, B. (2011). An extension of the theory of planned behavior to predict immediate hedonic behaviors and distal benefit behaviors. Food Quality and Preference, $22(7), 638-646$. 
Conner, M., Kirk, S. F. L., Cade, J. E., \& Barrett, J. H. (2001). Why do women use dietary supplements? The use of the theory of planned behaviour to explore beliefs about their use. Social science \& medicine, 52(4), 621-633.

Conner, M., Norman, P., \& Bell, R. (2002). The Theory of Planned Behavior and Healthy Eating. Health Psychology, 21(2), 194-201.

Erdman, J. W., Macdonald, I. A., \& Zeisel, S. H. (Eds.). (2012). Present Knowledge in Nutrition, Tenth Edition. Oxford, UK: Wiley-Blackwell.

Fila, S., \& Smith, C. (2006). Applying the Theory of Planned Behavior to healthy eating behaviors in urban Native American youth. International Journal of Behavioral Nutrition and Physical Activity, 3(1), 11.

Gajre, N., Fernandez, S., Balakrishna, N., \& Vazir, S. (2010). Breakfast eating habit and its influence on attention-concentration, immediate memory and school achievement. Indian Pediatrics, 45(10), 824.

Haines, P. S., Guilkey, D. K., \& Popkin, B. (1996). Trends in Breakfast Consumption of US Adults Between 1965 and 1991. Journal of the American Dietetic Association, 96(5), 464-470.

Keski-Rahkonen, A., Kaprio, J., Rissanen, A., Virkkunen, M., \& Rose, R. J. (2003). Breakfast skipping and health-compromising behaviors in adolescents and adults. European journal of clinical nutrition, 57, 842-853.

Koechlin, E., Ody, C., \& Kouneiher, F. (2003). The architecture of cognitive control in the human prefrontal cortex. Science, 302, 1181-1185.

Kothe, E., Amaratunga, R., \& Mullan, B. (2011). Randomised controlled trial of a brief theorybased intervention promoting breakfast consumption. Appetite, 56(1), 148-155. 
Kothe, E., Mullan, B., \& Butow, P. (In Press). Promoting fruit and vegetable consumption: Modeling behaviour change using the theory of planned behaviour. Appetite.

Lattimore, P. J., \& Halford, J. C. G. (2003). Adolescence and the diet-dieting disparity: Healthy food choice or risky health behaviour? British journal of health psychology, 8(4), 451463.

Matkovic, V., Fontana, D., Tominac, C., Goel, P., \& Chesnut, C. (1990). Factors that influence peak bone mass formation: a study of calcium balance and the inheritance of bone mass in adolescent females. The American Journal of Clinical Nutrition, 52(5), 878-888.

Mullan, B., \& Singh, M. (2010). A systematic review of the quality, content and context of breakfast consumption. . Nutrition and Food Science, 40(1), 81-144.

Mullan, B., Wong, C., Kothe, E., \& MacCann, C. (2012). Predicting breakfast consumption: A comparison of the theory of planned behaviour and the health action process approach.

NIH Consensus Panel. (1994). NIH Consensus Conference. Optimal calcium intake. NIH consensus development panel on optimal calcium intake. JAMA, 272, 1942-1948.

Norman, P., \& Conner, M. (1996). The role of social cognition models in predicting health behaviours: Future directions.

Rampersaud, G. C., Pereira, M. A., Girard, B. L., Adams, J., \& Metzl, J. D. (2005). Breakfast Habits, Nutritional Status, Body Weight, and Academic Performance in Children and Adolescents. Journal of the American Dietetic Association, 105(5), 743-760.

Sainsbury, K., \& Mullan, B. (2011). Measuring beliefs about gluten free diet adherence in adult coeliac disease using the theory of planned behaviour. Appetite, 56(2), 476-483.

Schwarzer, R., Schuz, B., Ziegelmann, J. P., Lippke, S., Luszczynska, A., \& Scholz, U. (2007). Adoption and Maintenance of Four Health Behaviors: Theory-Guided Longitudinal 
Studies on Dental Flossing, Seat Belt Use, Dietary Behavior, and Physical Activity. Annals of behavioral medicine, 33(2 ), 156-166.

Schwarzer, R., Sniehotta, F. F., Lippke, S., Luszczynska, A., Scholz, U., Schüz, B., et al. (2003). On the Assessment and Analysis of Variables in the Health Action Process Approach: Conducting an Investigation.

Seo, H.-S., Lee, S.-K., \& Nam, S. (2011). Factors influencing fast food consumption behaviors of middle-school students in Seoul: an application of theory of planned behaviors. Nutrition research and practice, 5(2), 169-178.

Shaw, M. E. (1998). Adolescent breakfast skipping: an Australian study. Adolescence, 33(132), $851-861$.

Verbeke, W., \& Vackier, I. (2005). Individual determinants of fish consumption: application of the theory of planned behaviour. Appetite, 44(1), 67-82.

Webb, T. L., \& Sheeran, P. (2006). Does changing behavioral intentions engender behavior change? A meta-analysis of the experimental evidence. Psychological Bulletin, 132(2), 249.

Wesnes, K. A., Pincock, C., Richardson, D., Helm, G., \& Hails, S. (2003). Breakfast reduces declines in attention and memory over the morning in schoolchildren. Appetite, 41(3), $329-331$.

White, K. M., Terry, D. J., Troup, C., Rempel, L. A., \& Norman, P. (2010). Predicting the consumption of foods low in saturated fats among people diagnosed with Type 2 diabetes and cardiovascular disease. The role of planning in the theory of planned behaviour. Appetite, 55(2), 348-354. 
Williams, P. (2005). Breakfast and the diets of Australian adults: An analysis of data from the 1995 National Nutrition Survey. International Journal of Food Sciences and Nutrition, $56(1), 65-79$.

Wong, C., \& Mullan, B. (2009). Predicting Breakfast Consumption: An application of the Theory of Planned Behaviour and the Investigation of Past Behaviour and Executive Function. British journal of health psychology, 14(3), 489-504. 
Table 1

Demographics and breakfast eating patterns

$$
\text { UK (\%) Australia (\%) }
$$

Gender

Males

20

36

Females

80

64

Ethnicity

Caucasian

74

70

Asian

9

2

Black/African

.5

3

Middle Eastern

1

2

Other

15.5

23

Breakfast consumption frequency

7 times a week

60

5-6 times a week

19

21

3-4 times a week

12

15

1-2 times a week

7

12

Never

3

9 
Table 2

Test of Between Subjects Effects by Country and Gender

\begin{tabular}{|c|c|c|}
\hline Country & $f$ & $p$ \\
\hline Relative Risk & 27.243 & $<.001$ \\
\hline Absolute Risk & 32.949 & $<.001$ \\
\hline Risk Severity & .758 & .384 \\
\hline Attitude & 22.059 & $<.001$ \\
\hline Subjective Norm & 17.584 & $<.001$ \\
\hline $\mathrm{PBC}$ & 6.816 & .009 \\
\hline Intention & 15.454 & $<.001$ \\
\hline Past Behaviour & 23.470 & $<.001$ \\
\hline Age & 43.727 & $<.001$ \\
\hline \multicolumn{3}{|l|}{ Gender } \\
\hline Relative Risk & .310 & .578 \\
\hline Absolute Risk & .006 & .936 \\
\hline Risk Severity & 6.534 & .011 \\
\hline Attitude & 2.225 & .136 \\
\hline Subjective Norm & 4.667 & .031 \\
\hline $\mathrm{PBC}$ & 3.250 & .072 \\
\hline Intention & 5.288 & .022 \\
\hline Past Behaviour & 4.483 & .035 \\
\hline Age & 122.572 & $<.001$ \\
\hline
\end{tabular}


Table 3

Pearson's correlation between study variables

\begin{tabular}{|c|c|c|c|c|c|c|c|c|}
\hline & RR & RA & $\mathrm{RS}$ & Attitude & $\begin{array}{l}\text { Subj. } \\
\text { Norm }\end{array}$ & PBC & Intention & Past Beh. \\
\hline Age & .051 & .053 & $-.088^{*}$ & -.051 & .038 & .019 & -.042 & -.034 \\
\hline RR & - & $.645^{* *}$ & $.318^{* *}$ & $.349^{* *}$ & $.293^{* *}$ & $.322 * *$ & $.348^{* *}$ & $.287^{* *}$ \\
\hline RA & - & - & $.294^{* *}$ & $.388^{* *}$ & $.340 * *$ & $.357 * *$ & $.397^{* *}$ & $.310^{* *}$ \\
\hline RS & - & - & - & $.134^{* *}$ & .066 & .073 & $.110^{* *}$ & $.085^{*}$ \\
\hline Attitude & - & - & - & - & $.349^{* *}$ & $.475^{* *}$ & $.524^{* *}$ & $.439 * *$ \\
\hline $\begin{array}{l}\text { Subj. } \\
\text { Norm }\end{array}$ & - & - & - & - & - & $.373^{* *}$ & $.401^{* *}$ & $.293^{* *}$ \\
\hline PBC & - & - & - & - & - & - & $.575^{* *}$ & $.484^{* *}$ \\
\hline Intention & - & - & - & - & - & - & - & $.751^{* *}$ \\
\hline
\end{tabular}

Note $. \mathrm{RR}=$ relative risk, $\mathrm{RA}=$ absolute risk, $\mathrm{RS}=$ risk severity, Subj. Norm= subjective norm, $\mathrm{PBC}=$ perceived behavioural control, Past $\mathrm{Beh}=$ past behaviour. $*$ Denotes correlations that are significant at the .05 level. ** Denotes correlations that are significant at the .01 level 
Table 4.

Factor analysis of Theory of Planned Behaviour and risk items

\begin{tabular}{|c|c|c|c|c|c|}
\hline \multirow[b]{2}{*}{ Item } & \multicolumn{5}{|c|}{ Component } \\
\hline & 1 & 2 & 3 & 4 & 5 \\
\hline Relative Risk 1 & .113 & .815 & .371 & .152 & .066 \\
\hline Relative Risk 2 & .133 & .797 & .402 & .154 & .065 \\
\hline Relative Risk 3 & .178 & .771 & .339 & .179 & .074 \\
\hline Absolute Risk 1 & .158 & .324 & .812 & .108 & .138 \\
\hline Absolute Risk 2 & .175 & .329 & .818 & .113 & .153 \\
\hline Absolute Risk 3 & .228 & .282 & .724 & .182 & .099 \\
\hline Risk Severity 1 & .047 & .095 & .064 & .918 & -.017 \\
\hline Risk Severity 2 & .030 & .106 & .114 & .913 & .016 \\
\hline Risk Severity 3 & .071 & .138 & .125 & .879 & .032 \\
\hline Attitudes 1 & .865 & .087 & .112 & .033 & .067 \\
\hline Attitudes 2 & .835 & .157 & .142 & .041 & .118 \\
\hline Attitudes 3 & .815 & .248 & .001 & .065 & .117 \\
\hline Attitudes 4 & .804 & .223 & .053 & .044 & .151 \\
\hline Attitudes 5 & .823 & -.058 & .246 & .021 & .132 \\
\hline Attitudes 6 & .828 & -.046 & .262 & .061 & .102 \\
\hline PBC 1 & .433 & .400 & -.012 & .008 & .573 \\
\hline PBC 2 & .084 & -.016 & .153 & -.012 & .840 \\
\hline PBC 3 & .046 & -.039 & .169 & .070 & .869 \\
\hline PBC 4 & .429 & .382 & -.105 & -.034 & .609 \\
\hline Subjective Norm & .276 & .122 & .316 & -.026 & .364 \\
\hline
\end{tabular}


Notes. $\mathrm{PBC}=$ perceived behavioural control. Extraction Method: Principal Component Analysis. Rotation Method: Varimax with Kaiser Normalization. Weightings above .5 are bolded 
Table 5

Hierarchical regression analysis: TPB variables and risk predicting intention

\begin{tabular}{|c|c|c|c|c|c|}
\hline & Variable & $\beta$ & $t$ & $p$ & $\mathrm{R}^{2}$ \\
\hline \multirow[t]{2}{*}{ Step 1} & Gender & -.187 & -4.65 & $<.001$ & \\
\hline & Country & -.150 & -3.75 & $<.001$ & .047 \\
\hline \multirow[t]{6}{*}{ Step 2} & Gender & -.055 & -1.70 & .090 & \\
\hline & Country & -.094 & -2.95 & $<.001$ & \\
\hline & Attitude & .272 & 7.51 & $<.001$ & \\
\hline & SN & .175 & 5.07 & $<.001$ & \\
\hline & PBC & .349 & 9.60 & $<.001$ & \\
\hline & & & & & .422 \\
\hline \multirow[t]{8}{*}{ Step 3} & Gender & -.038 & -1.18 & .238 & \\
\hline & Country & -.094 & -2.95 & .003 & \\
\hline & Attitude & .242 & 6.54 & $<.001$ & \\
\hline & SN & .153 & 4.41 & $<.001$ & \\
\hline & PBC & .325 & 8.88 & $<.001$ & \\
\hline & AR & .088 & 2.08 & .038 & \\
\hline & $\mathrm{RR}$ & .053 & 1.28 & .202 & \\
\hline & $\mathrm{RS}$ & -.005 & -.149 & .882 & .434 \\
\hline
\end{tabular}

Note: Gender ( $0=$ male, $1=$ female); Country $(0=$ Australia, $1=\mathrm{UK})$; DV=intention, $\mathrm{SN}=$ subjective norm; $\mathrm{PBC}=$ perceived behavioural control, $\mathrm{RA}=$ risk absolute; $\mathrm{RR}=$ relative risk; $\mathrm{RS}=$ risk severity 
Table 6

Hierarchical regression: TPB variables predicting behaviour

\begin{tabular}{llcccc}
\hline & Variable & $\beta$ & $t$ & $p$ & $\mathrm{R}^{2}$ \\
\hline Step 1 & Gender & -.144 & -3.60 & $<.001$ & \\
& Country & -.218 & -5.47 & $<.001$ & .057 \\
& Gender & -.031 & -1.13 & .259 & .004 \\
& Country & -.078 & -2.87 & $<.001$ & \\
& Intention 2 & .698 & 21.76 & .026 & .578 \\
& PBC & .071 & 2.23 & & \\
& & & & & \\
\end{tabular}

